\title{
INDUSTRY TRENDS TO 2040
}

\author{
Eckert, Claudia (1); Isaksson, Ola (2); Hallstedt, Sophie (3); Malmqvist, Johan (2); Öhrwall \\ Rönnbäck, Anna (4); Panarotto, Massimo (2)
}

1: The Open University; 2: Chalmers University of Technology; 3: Blekinge Institute of Technology; 4: Luleå University of Technology

\begin{abstract}
The engineering design community needs to development tools and methods now to support emerging technological and societal trends. While many forecasts exist for technological and societal changes, this paper reports on the findings of a workshop, which addressed trends in engineering design to 2040 . The paper summarises the key findings from the six themes of the workshop: societal trends, ways of working, lifelong learning, technology, modelling and simulation and digitisation; and points to the challenge of understanding how these trends affect each other
\end{abstract}

Keywords: Trends, Societal consequences, Design process, Systems Engineering (SE)

\section{Contact:}

Eckert, Claudia

The Open University

Engineering and Innovation

United Kingdom

c.m.eckert@open.ac.uk 


\section{INTRODUCTION}

We live in a rapidly changing world. Many of the technology trends that we are observing at the moment, like additive manufacturing or AI have been around since the 1990s, however it is taking industry a long time to make full use of their potential. This is partly to due to maturity and affordability of the technology, but also because engineering did not have the tools and methods to design with new technologies. To support the uptake of technology in the future, we need to develop tools and methods in parallel with the technologies and changing ways of working. Some of the most influential technologies of the coming 20 years are already on the horizon. Many forecasts exist for technological and societal changes, but the practical implications of engineering design are usually not articulated. This paper reports on findings from a workshop with experts from industry and academia. The workshop addressed trends in product development and engineering design practise to meet the needs and expectations in 2040. The year 2040 has been selected as it represents the future where many products currently designed will be in service.

\section{TREND FORCASTS FOR TECHNOLOGY AND SOCIETAL CHANGES}

Many forecasts exist for technological and societal changes that we are facing over a similar time scale, however few have looked in detail what the likely trends and challenges are for product development. Challenges are addressed on international level and expressed in visions and agendas such as the 17 UN goals for sustainable development until 2030 (UN, 2015), in industrial sections such a Europe's Vision for Aviation for 2050 (EU, 2011) and to meet technological shifts, such as Europe's "digital single market" (EU, 2015). Such international agendas further influence national agendas and financial instruments for R\&D as a means to drive change. Trends are being reported by national and international associations, tech watch organisations within their domains of interest, such as the Design Council in UK, and the international INCOSE for Systems Engineering.

The large societal trends of sustainability, servitization circular economy, population growth, and quality of life are influencing nearly everything in society. Dynamics in political, economic and climate/environmental situations have impact the conditions of everyday life. What is possible to predict by 2040 is difficult, yet demographic development is one relatively robust indicator.

An unsustainable society with less natural resources to share, with tougher competition, and with harder legislation will give less room of opportunities. Evidence of this development is presented in e.g., the latest IPCC's report "Global Warming of $1,5^{\circ} \mathrm{C}$ " (IPCC, 2018). Even if this report gives warning signals and urgency messages of clear benefits to people and natural ecosystems if the global warming is limited to $1,5^{\circ} \mathrm{C}$ instead of $2^{\circ} \mathrm{C}$, it also gives hope for possibilities to ensure a more sustainable and equitable society. At the same time, there is a high risk that global society will not make this transition to a more sustainable society in time and the tipping point is reached with no possibility to reverse it to a normal stage (Steffen et al., 2015). To avoid this situation there is a need to rethink more radically than we hitherto have been prepared to do.

One overarching technology trend is the current shift into a digital society is influencing broadly the society and industry through Industry 4.0 initiatives (Oztemel et al., 2018), that since introduced 2014 has gained widespread recognition as a trend. This drive the exploitation of autonomous, connected (IoT) products. Other types of trends include advancements in materials and manufacturing technologies through e.g. so called 3D printing that at present is focussed also for metallic applications. Technologies for energy generation, storage and distribution are emerging, where the carbon free society opens for other means of managing industries. Entrepreneurs are harvesting opportunities, whilst established industries struggle with both maintain a balance between incremental and radical mode of developing.

These trends will have a huge impact on the day to day activities of product designers, who will require new skills as well as tools and methods in response. The engineer's ability to harness new technologies will be vital in addressing the societal challenges. 


\section{METHODOLOGY}

The overall research process is summarised in Figure 1. The research started with a study of the literature on trend prediction, as summarised in section 2. This revealed a clear gap in an analysis of future trends in the product development processes. While many researchers and engineers think about future trends regularly, it was felt that everybody needed time to reflect about the topic. It was therefore decided to start with interviews of experienced engineers which gave individuals the time to develop their thoughts. The participants were selected from the circle of industry contacts of the authors, and largely were former collaborators. This aim was to gain a wide spread over industry

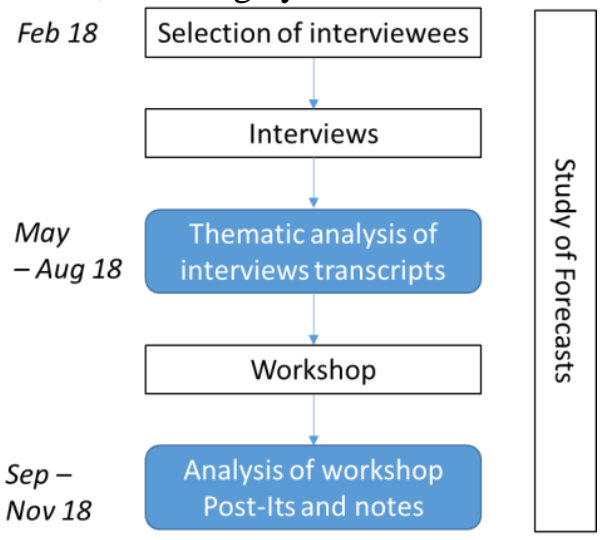
sectors and countries. A total of 6 in Sweden, 5 in the UK, 3 in Germany and 1 in Ireland were interviewed by either the first or the second author. The conversation with the participants centred around three open questions: "What trends do you foresee? What skills are required? What can universities do to support you in this?". The interviews were conducted in Swedish and in English. The English interviews were transcribed. Thematic analysis on the interviews revealed the following themes: transport, energy, product life cycle, system architecture, big data, simulation and engineering practise. The key points under these headings were summarised and send to the interviewees, as well as the workshop participants.

Figure 1. Overview of the research process

The workshop focused on the implications for product development. The workshop brought together over 40 experts from Swedish companies and international academics recruited through the research networks of the authors. The invitees were largely experienced engineers from large manufacturing companies in Sweden such as Volvo, SAAB, GKN, Astra Zeneca, SKF, NEVS, and many more with over 20 years of experience that the authors either knew or had been recommended by engineers they knew. The academics had a strong interest in engineering of complex products and were invited through the network of collaborators of the authors in the MMEP and Design Practise SIG. The summaries of the interviews were presented at the beginning of the workshop. The participants were also reminded of some technology ambitions that are already stated for 2040, such as the Norwegian initiative to have electric short distance flight by 2040 . To set the context of a changing world the workshop was opened with a key note by one of the academic contributors, who addressed both the role engineering has to play in creating a sustainable future, but also outlines the sustainability challenge we are facing through issues such as climate change, pollution, depletion of resources and a changing population.

The participants were grouped into mixed academic and industry groups with the spread of sectors and personalities. The groups were rotated through thematic stations every 30 minutes. The themes focussed on common issues for product development found in the interviews. The technical themes addressed technology in general, digitalisation and modelling and simulation. Engineering practise was covered under ways of working. Life-long learning covering educations and acknowledged the need of continues education. Society aimed to facilitating a discussion of trends that affected the designers and users of their products. Each theme was led by one of the authors who stayed with the theme throughout the day and facilitated an open discussion in the group. The trends were captured through anonymous postIt notes, which were added to a board, as well as notes from the station leaders.

\section{FUTURE TRENDS}

\subsection{Societal trends}

The room of opportunities for companies will decrease with time as long as the society is moving in an unsustainable direction (Robèrt and Broman, 2017). As societal trends are comparatively well articulated, the participants expressed five areas that required a global response:

- Social and administrative responsibilities: The expert group saw a need for the authorities to better express clearly what is required. Business models should be established on a national level to accelerate adaption and implementation of more sustainable products and services. In this way, 
social institutions and industry can meet the future goals together. In addition, common global legislations, incentives and nudging for new more sustainable solutions are needed to meet the societal challenges on the future market.

- Support to reach successful product development with future societal challenges. They perceived a need for support tools that can increase the knowledge of the engineering teams in the early design phases regarding the product's lifecycle implications, including information about material and energy issues. There were also suggestions of tools and models (simulationand visualisation models) that can predict sustainability impact and circularity options by creating scenarios regarding product usage and social behaviour, societal needs, and policy changes. To quantify sustainability, to monetize sustainability, to asses social sustainability and to move from performance-based product requirements to impact based product requirement is also needed.

- New development constellations: There is a need for more multidisciplinary approaches and a collaboration within the value chain from local to global actors but also between different stakeholders e.g. company, authorities and municipality. To share information, knowledge and to build capabilities across borders is therefore required.

- Long-term solutions - describing the future customer needs in a circular society: product resilience over time, multipurpose -and modular products to be reused and maintained. Products with sustainability scores and soft digital certification standards to guide the customers of their choices.

- Education: For engineers require knowledge and skills in the area of sustainability and engineering.

The expert groups highlighted a clear need for tools, methods and models that can predict, simulate, and visualise sustainability impact and circularity options. Scenario creation regarding product usage, social behaviours, societal needs, and policy changes are also of importance. The engineers looked for ways to quantify and monetize sustainability as well as to asses social sustainability to enhance the decisions in product development. Support is also needed for how to move from performance-based product requirements to sustainability impact-based product requirement.

\subsection{Ways of working}

The "ways of working" station addressed work practises in 2040. The ways of working are obviously affected by the general technology trends, which affect what we will be working on the digitalisation and modelling trends that determine what tools we have available for our work. However, some trends focussed on the way of working:

- End of highly prescribed processes: many felt that traditional gateway processes are too rigid and will be replaced by a more flexible approach to working. While several engineers mentioned agile scrum as an alternative to rigid processes, there was no expectation that agile will be the prevailing paradigm in 2040, even though many talked about working in intense scrums in the future.

- Integration of disciplines: as the products are becoming more multidisciplinary, engineers from different disciplinary backgrounds and experts from outside of engineering will have to be working together more closely. Many participants voiced a need for better system thinking skills in engineering processes, which will enable system integrators to bring in the contribution to highly specialised experts.

- Flexible working around co-located core teams: The participants felt that a stable core team will remain permanently located at central company sights, but they will be supported by a range of experts with specific skills, who will be hired for specific tasks and can be located anywhere. This could lead to a bifurcation of working practises with high standards for permanent employees and a gig economy for others.

- More diverse teams: Diversity will increase not only in disciplinary background, but also in ethnicity and location as remote working will become more accepted. The participants did on the whole not expect gender balance in engineering, as recent trends in undergraduate populations in core engineering discipline do not show a strong upwards trend. However, the number of women in less conventionally technical roles might increase.

- Collaboration with robots and AI systems: will increase and will be considered normal.

This raised interesting challenges for the product development community. The increased diversity in cultural and disciplinary background increase the need for effective and joint-up tools and methods to 
integrate across different disciplines. It also places a great emphasis on design representations that can be shared across different groups, which implies the need for a certain level of standardisation as well as increased literacy. The increased use of experts outside companies also have challenging implications for the protection of intellectual property.

\subsection{Lifelong learning}

The "lifelong learning" station addressed continuous learning and how engineers can stay updated throughout the professional career. This is increasingly important with the fast-developing technologies in industry, and the consequences this have on human behaviour, as well as increased awareness of engineering work on sustainability and ethics. The workshop discussions involved more the forms for how this can be achieved than the content. Some suggestions from the workshop were:

- Technology Innovation Conferences: This is not a new concept, but something that larger tech firms have applied over the years "a very good experience for life-long learning so far, and is important to continue with".

- Specialist vs Generalist: The continuous generalist and specialist knowledge dichotomy were addressed, as being more important than ever with the even wider variety of fields required in future product development work, with ever more knowledge available per field. Companies' investments in deep specialist knowledge was highlighted as "the key to success" -in combination with specialists working together with generalists at the right timing.

- Big data for learning: With the big data opportunities tools need to be developed to first, collect data in a secure way, and second, to visualize this data in comprehensible ways, to increase understanding and minimize risks of misinterpretations. A critical regard as well as building trust in the results of simulations and data is needed.

- Learning with digital tools: Robots, AI systems, augmented, virtual and mixed reality (AR, VR, MR) will make it possible to move learning methods to new heights, in experimental ways. However, it is required that continuously developed specialist knowledge is built in to the new technology tools, and there is an apparent risk if too static and simple, already known knowledge, is the only basis for these tech learning tools.

- The role of universities: The societal trend of fast, and sometimes false, facts in social media was highlighted as a dangerous threat if spread to engineering work, i.e. it could lead to lost respect for deep specialist knowledge, if "go at intuition" is too much encouraged. Here, universities as platforms for knowledge and learning also for professionals along their career were suggested to reduce this risk. Open boundaries in the educational system with inflowoutflow between professionals of different skills, and personalized programs made available through digital solutions and online (internet based) learning, were suggested. The role of universities as neutral places and knowledge hubs for learning, across companies and industries, and regardless of commercial competition, was stressed as important in the future.

Some informal discussions among participants from larger companies touched upon a concern between HR (human relations) departments and product development, highlighting a risk of HR not being aware of the requirements of future knowledge needs. When (if) increasing power in corporate management is given to administration, compared to $R \& D$, deliberately or not (this could be an unplanned consequence of organization and actions, e.g. regulations of traveling and R\&D conference participation), there is a risk that competence needs for future engineering are not met. It was concluded that responsibility for lifelong learning lies with the individual but encouraging organizational structures and recourses for this need to be secured.

\subsection{Technology}

As the workshop focusses on the trends for engineering design, the discussion of the workshop participants focusses on the technologies that supported design and manufacturing rather than the technology that are incorporated in their products. They highlighted the following trends:

- Artificial Intelligence and Augmented Reality as big trends in the technological landscape.

- The increase in computational power - enabled by novel computing methods - is going to make $\mathrm{AI}$ and AR more realistic scenarios for product development. Quantum computing was stressed as a ground-breaking technology in this context. Quantum computing will enable to run all product analyses in parallel (increasing computational power immensely), yet will increase the difficulty to 
understand the results made out of quantum computing. This is because the logic of quantum computing is substantially different than the human spatio-temporal logics.

- The increased connectivity among products throughout the whole lifecycle will increase personalization and automation, and the way individualized products will be developed. One participant pointed out: "if you want to understand needs, why running focus groups? You will ask sensors out in the field". The challenges in this context are related to cyber-security, and to identify the 'right' purpose of the data for the design activity: "there will be more people that do not want smart products that match their usage behaviours, but they would like to design their own products based on their data".

- The maturation and evolution of Additive Manufacturing (AM) will contribute to increase customisation and production on demand. Practitioners foresee AM as a "democratic technology". This will drive new business models and customer-manufacturer relationships. One participant pointed out: "what the company will sell to the customer is the knowledge to support the customer to design her own product, by suggesting what is doable and what is not".

- The uptake of new smart materials will drive new practices in design: "there will be materials that are gradually changing over time, so that we will not design and change the product structures, but we will design and change the material properties of the product".

- New energy sources will drive new design practices reducing consumption. The challenge in this context is to ensure the security of the supply.

The workshop highlighted a general consensus about the role of digitalization ("more value will be generated through software content"), yet recognized some 'contradictions' at the intersection of some technological and societal trends. Such contradictions will have profound implications for product development capabilities in 2040. For example, the raising trend of individualization is apparently conflicting with the raising trend of sharing economy: new practices will be needed to effectively individualize products that are actually going to be shared. At the same time, more technology will become available, in a market that might avoid new technology (either because of market saturation or because of a conscious choice to avoid consumption): "we need to be really smart to be able to offer technologies and products to people that are actually suspicious about using new technology".

\subsection{Modelling and simulation}

The importance and range of applications of modelling and simulation (M\&S) is continuously increasing. Over decades, $M \& S$ has been seen as a way to analyse and predict phenomena, that are too costly, complex, dangerous or time consuming to understand through studying real conditions. For product development $\mathrm{M} \& \mathrm{~S}$ enables an evaluation of the product before a physical prototype exists and can thereby support decision making. The participants identified the following trends:

- M\&S environments are becoming interlinked. Design decision making requires trading different aspects, often originating from different domains, off against each other. Tools for M\&S in domains (e.g. structural mechanics) have evolved within their domain, but the multidisciplinarily of the design problems drive also the need for interlinking different domains. The interlinking trend is justified by 1) that goodness of design decisions rely on trade-offs, and 2) the increased ability to manage, combine and aggregate both data and methods, also mixing measured data with digitally generated data

- M\&S of entire life cycles. Since following circular economy and service orientation, products increasingly include behaviour through life with increasing responsibility for manufacturers. This is expected to drive the development of new and enhanced M\&S tools for Design and Development support. This is further expected to support evolving designs, covering a range of life cycles to capture upgradeability and technology replacement strategies.

- M\&S Democratisation. M\&S has long been the tools for analysis specialists, whereas designers have had limited direct use of (advanced) M\&S tools. It is expected that more roles in product development have M\&S tools readily available "at their fingertips". Specialists are still needed, but increasingly for developing and validating the tools and their applicability and constraints.

- M\&S trust increased. It is anticipated that the increased sensing ability will (partly) be used to validate and train M\&S methods and tools. Decision making will need to rely on the M\&S results, from guiding to ruling. Trust need to increase, and data availability need to be used to achieve this. 
Multiple layers of M\&S, following the systems of systems logic, are emerging which might affect trust in $M \& S$ "systems of systems". M\&S is used to predict and understand ever more complex physical or social phenomena, which maintains "advisory" status of M\&S in decision making. Model validity remain an increased focus either way.

- Complete integrated digital twins. The recent (decade long) concept of digitally defined replicas - called Digital Twins" - of real products and processes continue to evolve, to include an even more complete representation of the existing reality. Advancements in sensing and computing power release many of the constraints from history. Digital Twins expect to evolve into variable designs behaviour in rich real situations.

- M\&S blending with AI in synthesis and analysis, along with the trends of the "exploding" capability to generate and measure data, the methods and tools to manage, visualise, interpret and operate on large and evolving datasets will bring AI closely integrated with M\&S tools and methods.

- M\&S becoming gamified. Both demographic effects and speed of ICT development drive towards a more instant response functionality of M\&S tools. Young people are brought up with "instant response" of queries. ICT and AI advances will enable swifter analysis of designs options. It is expected that designers 2040 will be able to assess impact of alternative designs "instantly" and that underlying computational power is used to validate such tools.

In summary, $M \& S$ methods and tools will be blended with design tools, readily available for designers and developers, better enabling instant interaction for decision makers. ICT and AI will be used to enable such design aids. The countertrends can be the increased diversity and depth of specific studies, where specialists are needed. The ability to predict behaviour of systems of systems in real situations remains a challenge and will challenge design researcher with $M \& S$ interests.

\subsection{Digitalisation}

Digitalisation has been on the agenda for development of engineering processes during the last 50 years. The expert group identified some emerging capabilities and challenges in the area, including:

- Product content. It was agreed that in the future "all" products will be cyber-physical systems, often based on an open architecture requiring and enabling both continuous updates and product development. Open product architectures will support re-programming and customization by users. Products will be "smart", with consciousness of space and time, as well as awareness of own state. Products will collect massive amounts of use data and feed that back for monitoring purposes as well as self-learning and -optimization.

- Working environments. Some experts argued that future working environments would assume a practice of working from anywhere, the home, on public transport, in the office etc. Digital engineering tools and AR/VR technology would need to support this practice. Other experts noted that the remote working trend may already have peaked and reversed, referring to IBM as an example. Personal digital assistants will be in place to structure and organize daily tasks.

- Data capturing, storage and use. With digital twin technologies supported by advanced sensor systems, massive amounts of use data can be captured. Storage is expected to be cheap, even "free". Data analytics will discover use patterns and drive adaptations of product individuals as well as feed back to product developers. Product developers will increasingly monitor the fleet of delivered products and continually launches fixes and upgrades. The experts further pointed to challenges such as respect for personal integrity, security and long-term data format robustness.

- Artificial intelligence. AI was described as a future co-worker, helping with, for example, debugging and natural language-based programming. Still, there was no consensus of the impact of AI on creative or decision-making tasks. Some experts maintained that AI will be helpful in "reducing waste", but not for creative work. Others experts pointed out that AI techniques such as reinforcement learning have already been demonstrated to be able to carry out creative tasks, such as composing music.

- Virtual validation and verification. Future digital tools and computer hardware will enable many more iterations and thus verification and optimization of product degree. The advances are particularly strong within disciplines, the classic "islands of automation" challenge. For validation and verification of future even more complex cyber-physical systems, more powerful tools for integrated multidisciplinary simulation are needed. Virtual validation of user experience also 
remains a challenging issue. AR/VR technology may address some aspects, much there is much work ahead.

\section{OBSERVATION AND FURTHER WORK}

There was amount of consensus among the participants about the expected trends. They noticed this themselves and suggested that this might be due to the group being fairly homogenous, i.e. middle engineers from large companies. While the participants were aware that the trends would affect each other, they discuss few of the influences trends would have on each other. However, the same issue occurred at the different topic stations, for example the need for lifelong learning was brought up both in the ways of working and the societal trends. Digitalisation also run as a topic through all of the stations from on line learning environment to virtual collaboration and the increased simulation.

It was difficult for the participants to think through the trends over a 20 year time scale. The trends that they foresaw where related to current working practise in two ways:

- Amplification of trends or practises that have already started. They identified recent changes that they expected to stay and increase over the next years, for example remote and virtual working has become to be used seriously in industry and they suggested that this would be a big trend.

- Solutions to the problems they are facing today were expected as trends for the future. For example, the integration of hardware and software solutions is a huge issue in contemporary industry and they assumed that a solution would be found for that.

All participants agreed that we live at present in politically and socially highly uncertain times, which will have an influence on engineering design in multiple. In the light of multiple potential future scenarios, which they acknowledged, most people assumed for the purpose of looking at the future of engineering design that the situation would roughly stay the same. This had the effect that the Swedish engineers and academic largely saw the change to digital future as a huge opportunity, which the companies need to embrace. By contrast the British interviewees and academics had a much more dystopian outlook and were seriously concerned about the prospect of engineering design in the UK.

Two particular trends were brought up in all interviews and most of the group sessions:

- The need for more system thinkers, who can handle the increased complexity and integration. This was complemented by the need for deep subject expertise.

- Mathematical, Statistics and Programming skills will be increasingly important as experts who are brought into engineering design teams, but also that designers and managers

The outcomes of the workshop will be analysed further and collated into a report which will be circulated to the participants. The authors are also working on articulating the implications for engineering education and design research.

\section{REFERENCES}

EU. (2011), "Fligthpath 2050 - Europe's Vision for Aviation", Publications Office of the European Union. ISBN 978-92-79-19724-6 https://ec.europa.eu/transport/sites/transport/files/modes/air/doc/flightpath2050.pdf

Hallstedt S. and Isaksson O. (2017), "Material criticality assessment in early phases of sustainable product development", Journal of Cleaner Production, Vol. 161, pp. 40-52.

"Intergovernmental Panel on Climate Change -IPCC (2018) Global warming of $1,5^{\circ} \mathrm{C}$ ", https://www.ipcc.ch/site/assets/uploads/sites/2/2018/07/SR15_SPM_version_stand_alone_LR.pdf

Oztemel, E. and Gursev, S. J. (2018), "Literature review of Industry 4.0 and related technologies", Intell Manuf. https://doi.org/10.1007/s10845-018-1433-8

Robèrt, K.H. and Broman, G. (2017), "Prisoners' dilemma misleads business and policy making”, Journal of Cleaner Production, Vol. 140, pp.10-16.

Steffen, et al. (2015), "Guiding human development on a changing planet", Science. Vol. 347, https://doi.org/10.1126/science.1259855

UN. (2015), "Transforming our world: the 2030 Agenda for Sustainable Development", https://www.un.org/sustainabledevelopment/development-agenda/

Vivanco, D. F., René K., and van der Voet, E. (2016), "How to deal with the rebound effect? A policy-oriented approach”, Energy Policy, Vol. 94, pp. 114-125.

\section{ACKNOWLEDGMENTS}

GoCo and Chalmers for hosting the workshop, Open University ESTEEM for funding the interviews. 